\title{
First step to observing gravitational waves
}

\author{
Designed with a laser metrology system, LISA Pathfinder is on track to demonstrate the first in-flight \\ test of low-frequency gravitational wave detection metrology in space.
}

\begin{abstract}
The European Space Agency's Laser Interferometer Space Antenna (LISA) Pathfinder blasted off from the French Guiana at 04:04 GMT on 3 December 2015. It is on a technology demonstration mission for future spaceborne gravitational wave observatories that would put Einstein's general theory of relativity to the test. All of the major noise sources in a gravitational wave detector are local to one spacecraft; the added noise coming from laser light traversing 5 million $\mathrm{km}$ through interplanetary space, for example interaction with interplanetary plasma, is negligible.

"We essentially shrink the 5 million $\mathrm{km}$
\end{abstract} arm of LISA to $38 \mathrm{~cm}$ and locate both ends of the arm inside one spacecraft. This way, we are susceptible to most of the noise budget terms of LISA and some extra terms not seen in LISA, but without the expense of the million kilometres, multi-spacecraft instrument," said Paul McNamara,

LISA Pathfinder Project Scientist, who also emphasized that LISA is not a gravitational wave detector, but testing the technology.

For LISA, the effect of a passing gravitational wave is to change the proper distance between two inertial points in space. In LISA Pathfinder, the inertial points are two free-floating gold-platinum test masses. Assuming the test masses are free from all forces bar gravity, when a gravitational wave passes, the distance between them, as measured by light, will change. This is the change LISA measures. "It sounds simple, but the devil is in the detail," McNamara stressed.

Assuming an average gravitational wave passes the Earth, the diameter of the Earth will change by less than the size of a proton, because $h=\mathrm{d} L / L$, where $h$ is the strain caused by passage of a gravitational wave $\left(1 \mathrm{e}^{-23}\right)$ and $\mathrm{d} L / L$ is the fractional length change of the detector. "Therefore, we need a large detector, and a very sensitive laser interferometric read-out system. We will test the latter with LISA." In practice, what LISA measures is the relative length change of two arms. This way, it can suppress effects such as laser frequency noise; think of a Michelson interferometer with arms of equal length.

One may be interested in knowing what kind of optics are involved in LISA. McNamara told Nature Photonics that it consists of a 1,064 nm Nd:YAG nonplanar

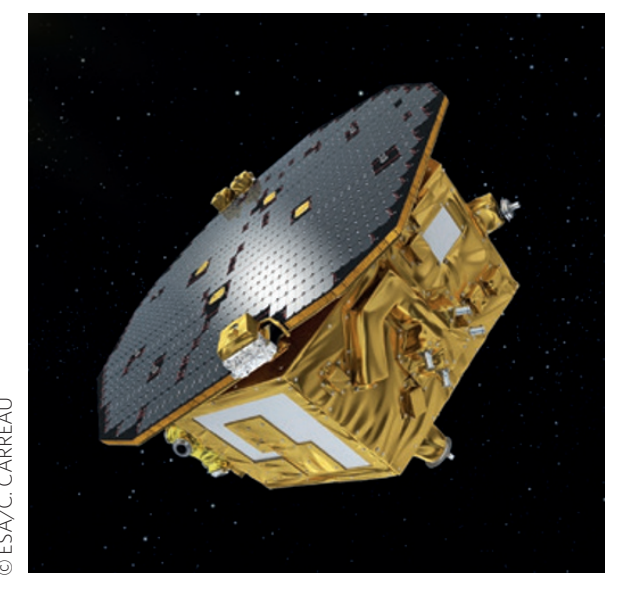

ring oscillator with about $40 \mathrm{~mW}$ output power. The light is split into two paths; each passes through an acousto-optic modulator, creating a frequency difference between the beams of $\sim 1 \mathrm{kHz}$ - the heterodyne frequency. The light is then transported via single-mode, polarizationmaintaining fibres to a monolithic Zerodur optical bench with fused silica mirrors and beamsplitters that are bound to the surface with hydroxy-catalysis bonds. There are four Mach-Zehnder interferometers on the optical bench - three with equal arms, and one with a deliberate arm-length mismatch. The first interferometer with equal arms is used to measure the displacement of one test mass with respect to the optical bench that is rigidly mounted to the spacecraft. The second is used to measure the differential displacement of the two free-floating test masses that have no mechanical contact to spacecraft - this is the main science channel of the mission. The third is a reference interferometer that allows LISA to remove any common mode noise imparted on the light before it reaches the optical bench. The interferometer with unequal arms is used to measure the frequency noise of the laser to stabilize the laser frequency. The interference signals are recorded using InGaAs quadrant photodiodes. There are two quadrant photodiodes per interferometer at each port of the combining beamsplitter to provide for redundancy. The phase measurement is read out using a single-bin discrete Fourier transform algorithm.

"We will demonstrate the free fall (residual acceleration with reference to inertial space) of the test masses at a level required by a large-scale observatory such as LISA. The true output of LISA Pathfinder is not a set of blueprints for LISA, but is a detailed physical model of the forces acting on the test masses in a space environment, which is inside a spacecraft," said McNamara.

According to McNamara, LISA Pathfinder's payload commissioning starts on 11 January 2016 and will last until 29 February 2016. On 1 March 2016, science operations will begin and the system will be optimized to demonstrate the differential acceleration requirements. This involves running a series of experiments and investigations to allow each major term in the noise budget to be probed, thereby linking the physical model with on-orbit results. The LISA Pathfinder science phase is essentially a long, very detailed commissioning phase of the LISA instrument. "We will learn a lot from it, which will not only allow us to optimize the design of LISA, but also to optimize the operations of the observatory," said McNamara, who added that the first results will be available around May 2016. With the results from LISA Pathfinder, the science community and the European Space Agency (ESA) will be in a position to propose the next step - a fullsized LISA-like observatory.

Given such a big task, one can't help but wonder how the international team of scientists works together. "Extremely well!" said McNamara delightfully. "Most of us have worked previously on the ground-based detectors (GEO600, LIGO and VIRGO). It was not a completely new collaboration. Also, LISA is one instrument - there is no competition between principal investigators on whose camera gets to observe at a particular time or orbit. For LISA Pathfinder and LISA to be successful, all of the subsystem units work together, and the team must also work together. Luckily for me, and [the] ESA in general, our science team is a joy to work with. Over the years we have become one very cohesive team, not a set of individuals from various institutes around Europe."

It's not an overstatement that LISA Pathfinder is the first mission of its kind. The path from the original concept to the production of flight hardware has not been easy. It is now time for harvesting. 